Cite this: Phys. Chem. Chem. Phys., 2013,

\title{
Nuclear magnetic resonance study of ion adsorption on microporous carbide-derived carbon $†$
}

15, 7722

\author{
Alexander C. Forse, ${ }^{a}$ John M. Griffin, ${ }^{a}$ Hao Wang, ${ }^{\text {ab }}$ Nicole M. Trease, ${ }^{b}$ \\ Volker Presser, ${ }^{c}$ Yury Gogotsi, ${ }^{d}$ Patrice Simon ${ }^{\mathrm{e}}$ and Clare P. Grey ${ }^{* a b}$
}

Received 20th March 2013, Accepted 21st March 2013

DOI: $10.1039 / \mathrm{c} 3 \mathrm{cp} 51210 \mathrm{j}$

www.rsc.org/pccp

\begin{abstract}
A detailed understanding of ion adsorption within porous carbon is key to the design and improvement of electric double-layer capacitors, more commonly known as supercapacitors. In this work nuclear magnetic resonance (NMR) spectroscopy is used to study ion adsorption in porous carbide-derived carbons. These predominantly microporous materials have a tuneable pore size which enables a systematic study of the effect of pore size on ion adsorption. Multinuclear NMR experiments performed on the electrolyte anions and cations reveal two main environments inside the carbon. In-pore ions (observed at low frequencies) are adsorbed inside the pores, whilst ex-pore ions (observed at higher frequencies) are not adsorbed and are in large reservoirs of electrolyte between carbon particles. All our experiments were carried out in the absence of an applied electrical potential in order to assess the mechanisms related to ion adsorption without the contribution of electrosorption. Our results indicate similar adsorption behaviour for anions and cations. Furthermore, we probe the effect of sample orientation, which is shown to have a marked effect on the NMR spectra. Finally, we show that $a{ }^{13} \mathrm{C} \rightarrow{ }^{1} \mathrm{H}$ cross polarisation experiment enables magnetisation transfer from the carbon architecture to the adsorbed species, allowing selective observation of the adsorbed ions and confirming our spectral assignments.
\end{abstract}

\section{Introduction}

Climate change and fossil fuel depletion are changing the world's attitude towards energy production and consumption. More renewable energy generation is required and technologies such as electric vehicles are being developed, both requiring the development of electrical energy storage devices such as batteries and electrical double-layer capacitors. Whilst batteries can store large amounts of energy for a given volume or mass, they suffer from limited cycle lives, restricted power handling capabilities and further limitations regarding safety

\footnotetext{
${ }^{a}$ Department of Chemistry, University of Cambridge, Lensfield Road, Cambridge, CB2 1EW, UK. E-mail: cpg27@cam.ac.uk

${ }^{b}$ Department of Chemistry, Stony Brook University, Stony Brook, NY 11794, USA

${ }^{c}$ INM - Leibniz-Institute for New Materials, Energy Materials Group, Campus D2 2, D-66123 Saarbrücken, Germany

${ }^{d}$ Department of Materials Science and Engineering and A.J. Drexel Nanotechnology Institute, Drexel University, Philadelphia, PA 19104, USA

${ }^{e}$ Université Paul Sabatier, CIRIMAT UMR CNRS 5085 Toulouse, 31062, France and Réseau sur le Stockage Electrochimique de l'Energie (RS2E), FR CNRS 3459,

France

$†$ Electronic supplementary information (ESI) available: SEM images and porosity analysis of carbons, spectral fits, derivation of formulae used to calculate area per anion, supporting NMR spectra and pulse sequences. See DOI: 10.1039/c3cp51210j
}

and temperature requirements. ${ }^{1}$ Consequently, alternative energy storage devices such as electrical double-layer capacitors, also known as ultracapacitors or supercapacitors, have attracted significant attention. Supercapacitors store charge by ion electrosorption, with charge in the electrode balanced by a layer of ions of opposite polarity in the electrolyte. ${ }^{2}$ This nonfaradaic storage mechanism allows rapid rates of charge and discharge and essentially unlimited cycle lives, making supercapacitors well suited to high power applications. ${ }^{3}$ Supercapacitors typically employ porous carbon electrodes as they have large surface areas for ion electrosorption, good electronic conductivities and relatively low production cost. ${ }^{4}$ In particular, activated carbons, derived from carbon-rich organic precursors such as coconut shells or wood, are the materials of choice in commercial devices. ${ }^{5}$ Such activated carbons exhibit pores with different diameters, often ranging from micropores (diameters less than $2 \mathrm{~nm}$ ), and mesopores (diameters between 2 and $50 \mathrm{~nm}$ ), to macropores (diameters greater than $50 \mathrm{~nm}$ ) according to the IUPAC classification. ${ }^{6}$

While a vast number of different carbons have been studied as supercapacitor electrode materials, ${ }^{4,6,7}$ carbide-derived carbons (CDCs), produced by chlorine treatment of metal carbides, have recently gained particular attention. ${ }^{8}$ CDCs have microporous 
structures with narrow pore size distributions. ${ }^{9,10}$ Control of the chlorination temperature used in the synthesis allows finetuning of the average pore size in the range 0.6 to $1.1 \mathrm{~nm}$, with higher temperatures resulting in a larger average pore size. ${ }^{8}$ Chmiola et al. reported an increase in the capacitance of titanium carbide-derived carbon (TiC-CDC) at pore sizes of less than 1 nanometre, using an electrolyte comprising tetraethylammonium tetrafluoroborate $\left(\mathrm{NEt}_{4} \mathrm{BF}_{4}\right)$ in acetonitrile. ${ }^{11}$ These results challenged the previous view that pores smaller than the solvated electrolyte ions do not contribute significantly to the capacitance. It was hypothesised that ion desolvation allowed a closer approach of charge centres at the electrode-electrolyte interface. ${ }^{12}$ Modelling and simulation studies have progressed the understanding of charge storage in microporous carbons, ${ }^{13-19}$ whilst new experimental methods have also been developed. ${ }^{20-24}$ Despite recent advances, further experimental techniques must be developed to directly probe the nature of ion adsorption and electrosorption in micropores.

In contrast to other techniques, nuclear magnetic resonance (NMR) spectroscopy allows the comprehensive investigation of the local structure and dynamics of electrode-electrolyte interfaces in a non-invasive and element selective way. ${ }^{25}$ This latter feature uniquely allows the separate observation of cations and anions in electrochemical systems. NMR has been used to study the adsorption of molecules on activated carbons, ${ }^{25-28}$ and the same techniques have been used to study adsorption inside carbon nanotubes (CNTs). ${ }^{29-32}$ It has been well established that resonances corresponding to molecules adsorbed on carbon surfaces show a shift to low frequencies (relative to the corresponding free species), making NMR a very useful tool to study ion adsorption. This approach has been extended to study the effect of an applied potential during the operation of supercapacitors with activated carbon electrodes. Ex situ experiments $^{33,34}$ (NMR spectra acquired on a dismantled supercapacitor after electrochemical cycling) can be combined with magic-angle spinning (MAS) techniques to improve spectral resolution. However, in situ experiments ${ }^{35}$ (NMR spectra acquired during cycling) are, in principle, preferable since they give a realistic picture of working devices.

Whilst previous experimental NMR studies have focused on adsorption on a variety of activated carbons and CNTs, a more systematic approach is desirable to obtain detailed insight into ion adsorption within carbon micropores. To study the poresize effect, here we focus our attention on CDCs with controlled pore sizes and narrow pore size distributions. NMR experiments allow the identification of in- and ex-pore ion environments. We carry out multinuclear NMR experiments to probe the adsorption of electrolyte cations and anions, shown to display very similar adsorption behaviour. We also explore some of the practical considerations relevant to envisaged in situ studies of supercapacitors, with sample orientation shown to be important for both interpreting spectra and improving resolution of features. Finally, a through-space magnetisation transfer experiment (from ${ }^{13} \mathrm{C}$ enriched carbon to the ${ }^{1} \mathrm{H}$ spins of the electrolyte cations) enables identification of species in close proximity to the carbon surface, providing conclusive evidence for our peak assignments.

\section{Experimental}

\section{Carbon materials}

TiC-CDC powder was synthesised by chlorine treatment of titanium carbide as described elsewhere in more detail. ${ }^{9}$ Commercial TiC (Alfa Aesar) with an average particle size of around $2 \mu \mathrm{m}$ was used as the precursor material. TiC powder was placed in a quartz glass boat and transferred to a quartz glass tube furnace. The sample was heated to the desired temperature $\left(600,800\right.$, and $\left.1000{ }^{\circ} \mathrm{C}\right)$ in an atmosphere of high purity argon gas, and the gas switched to dry chlorine for $3 \mathrm{~h}$. Subsequent to the chlorine treatment, the gas was changed to pure argon, and then to pure hydrogen, with the sample held at $600{ }^{\circ} \mathrm{C}$ for $2 \mathrm{~h}$ to remove chlorine and chloride residues. Finally, the sample was cooled to room temperature in high purity argon. Samples treated in chlorine gas at a temperature $X{ }^{\circ} \mathrm{C}$ (e.g. $\left.600{ }^{\circ} \mathrm{C}\right)$ are referred to as TiC-CDC- $X$ (e.g. TiC-CDC-600).

Isotopic $\mathrm{Ti}^{13} \mathrm{C}-\mathrm{CDC}-1000$ was synthesised as follows. A stoichiometric mixture of ${ }^{13} \mathrm{C}$ powder ( $0.4 \mathrm{~g}$, 99 at\%, Sigma Aldrich) was mixed with crystalline titanium $(1.7 \mathrm{~g}, \geq 99.99$ at $\%$, Sigma Aldrich; particle size: 5-10 $\mu \mathrm{m}$ ) in an agate mortar and then transferred to a graphite-plated alumina crucible. The resulting powder material was heated $\left(10 \mathrm{~K} \mathrm{~min}^{-1}\right)$ to $1550{ }^{\circ} \mathrm{C}$ in flowing argon under atmospheric pressure and held at that temperature for $12 \mathrm{~h}$. Finally, the sample was cooled down to room temperature $\left(10 \mathrm{~K} \mathrm{~min}^{-1}\right)$. The resulting monolithic material was ground by hand with an agate mortar to an average particle size between 2 and $50 \mu \mathrm{m}$ (see ESI, $†$ Fig. S1). X-ray diffraction confirms that the material consisted predominantly of microcrystalline TiC, while a small fraction $(<1 \mathrm{wt} \%)$ graphitic carbon (i.e., non-reacted precursor material) was found via Rietveld refinement. The titanium was extracted by treatment in dry chlorine. For this, the sample material was placed in a quartz glass boat, inserted in a tube furnace and flushed in Argon for $30 \mathrm{~min}$. The sample was heated $\left(10 \mathrm{~K} \mathrm{~min}^{-1}\right.$ ) to $1000{ }^{\circ} \mathrm{C}$ and then kept, for $3 \mathrm{~h}$ in flowing chlorine. The sample was then flushed with argon gas and treated in flowing hydrogen at $600{ }^{\circ} \mathrm{C}$ for $3 \mathrm{~h}$ to remove residual chlorine and chloride species. After a final argon flushing, the resulting CDC powder was cooled to room temperature.

Carbon films were fabricated using the standard method for preparing film electrodes, with a mixture of carbon powder (95 wt\%) and polytetrafluoroethylene (PTFE) binder (5 wt\%). More details on the electrode preparation can be found in, for example, ref. 11.

\section{Electrolytes}

Tetraethylammonium tetrafluoroborate, $\mathrm{NEt}_{4} \mathrm{BF}_{4}, \quad(\geq 99.0 \%$, Sigma Aldrich) $1.5 \mathrm{M}$ in acetonitrile, ACN, (99.8\%, Sigma Aldrich) was used in the majority of experiments, referred to as $\mathrm{NEt}_{4} \mathrm{BF}_{4} / \mathrm{ACN}$. In cases where ${ }^{1} \mathrm{H}$ NMR spectra were acquired, an electrolyte of the same salt and concentration was prepared using deuterated acetonitrile, $\mathrm{D}_{3} \mathrm{CCN}$ (99.80\%, Eurisotop). We refer to this electrolyte as $\mathrm{NEt}_{4} \mathrm{BF}_{4} / \mathrm{dACN}$. 


\section{NMR sample preparation}

3.1 Samples in bags. Carbon film pieces (6.0 mg, typically $8 \times 4 \times 0.25 \mathrm{~mm}$ ) were cut and heated for at least $15 \mathrm{~h}$ at $200{ }^{\circ} \mathrm{C}$ on a vacuum line to remove residual water, before being transferred to an argon glove box. A carbon film piece was placed inside a plastic bag (3M Scotchpak HB-P-69731). Having hermetically sealed the sample on three sides, the specified volume of electrolyte was micro-syringed onto the carbon before sealing the final side of the bag. Whilst the microsyringe allowed accurate measurement to within $0.5 \mu \mathrm{L}$ of the desired volume, at loading volumes above $10.0 \mu \mathrm{L}$ the carbon film became saturated and small amounts of electrolyte were lost on sealing. Samples in bags were wrapped in PTFE tape to fix their orientation in the NMR coil.

3.2 Samples in MAS rotors. A carbon film piece $(3.0 \mathrm{mg})$ was cut and heated for at least $15 \mathrm{~h}$ at $200{ }^{\circ} \mathrm{C}$ on a vacuum line before being transferred to an argon glove box. The film was cut into small pieces and approximately half of these were packed into a $2.5 \mathrm{~mm}$ outer diameter zirconia MAS rotor, before adding the specified volume of electrolyte by micro-syringe. The remaining carbon was then packed before capping the rotor (vespel cap). For electrolyte volumes above and including $7.5 \mu \mathrm{L}$, accurate control of the syringed volume was not possible due to electrolyte leakage.

\section{NMR}

NMR experiments were performed using Bruker Avance II spectrometers operating at magnetic field strengths of 7.05 and 9.4 T, corresponding to ${ }^{1} \mathrm{H}$ Larmor frequencies of 300.2 and 400.4 MHz, respectively. ${ }^{11} \mathrm{~B}$ NMR spectra of samples in bags were recorded using a Bruker single-channel static NMR probe fitted with a $6 \mathrm{~mm}$ diameter solenoid coil. ${ }^{19} \mathrm{~F}$ NMR spectra of samples in bags were recorded using a Chemagnetics singlechannel probe fitted with a $7 \mathrm{~mm}$ diameter solenoid coil. NMR spectra of samples contained within MAS rotors were obtained under static conditions using a Bruker $2.5 \mathrm{~mm}$ double resonance probe. Adsorption experiments were performed using a spin-echo pulse sequence $\left(90^{\circ}-\tau-180^{\circ}-\tau\right.$ - acquire) to avoid baseline distortions and to remove background signals associated with either the plastic bag or probe. Spin-echo $\tau$ delays of $50 \mu \mathrm{s}\left({ }^{11} \mathrm{~B}\right)$, and $100 \mu \mathrm{s}\left({ }^{19} \mathrm{~F}\right.$ and $\left.{ }^{1} \mathrm{H}\right)$ were used, except for ${ }^{19} \mathrm{~F}$ NMR spectra of samples in bags, where a slightly longer $\tau$ delay of $200 \mu \mathrm{s}$ was necessary to remove the stronger background signal associated with the PTFE resonance. Radiofrequency strengths of between $50-100 \mathrm{kHz}$ were used for all nuclei studied. For the ${ }^{13} \mathrm{C} \rightarrow{ }^{1} \mathrm{H}$ CP NMR experiment, transverse magnetisation on ${ }^{1} \mathrm{H}$ was obtained by cross-polarisation from ${ }^{13} \mathrm{C}$ using a contact pulse duration of $8 \mathrm{~ms}$ (ramped for ${ }^{1} \mathrm{H}$ ). A spin-echo with a $\tau$ delay of $100 \mu$ s was incorporated prior to acquisition of the FID, during which no heteronuclear decoupling was applied. ${ }^{11} \mathrm{~B}$ NMR spectra were referenced relative to boric acid solution $(0.1 \mathrm{M})$ at $19.6 \mathrm{ppm},{ }^{19} \mathrm{~F}$ NMR spectra were referenced relative to hexafluorobenzene liquid at $-164.9 \mathrm{ppm}$ and ${ }^{1} \mathrm{H}$ NMR spectra were referenced relative to tetramethylsilane using the $\mathrm{CH}_{3}$ resonance of liquid ethanol at $1.2 \mathrm{ppm}$ as a secondary reference. All liquid reference samples were contained within MAS rotors, which were either inserted directly into the MAS probe, or wrapped in PTFE tape for use in static NMR probes. Sufficiently long recycle delays were used to ensure that none of the ${ }^{1} \mathrm{H},{ }^{11} \mathrm{~B}$ and ${ }^{19} \mathrm{~F}$ signals were saturated, allowing for quantification of the different environments. Additional experimental details are given in the relevant figure caption.

\section{Results and discussion}

\section{1. ${ }^{11}$ B NMR study of anion adsorption on TiC-CDC-1000}

To investigate the adsorption of the $\mathrm{BF}_{4}$ anions, ${ }^{11} \mathrm{~B}$ NMR experiments were performed. Experiments were performed on samples in bags, which represent a model for supercapacitor electrodes. Spectra were acquired with samples in the horizontal orientation (Fig. 1a) as in our previous work. ${ }^{35}$ Fig. 1b shows ${ }^{11} \mathrm{~B}$ NMR spectra of samples in bags containing pieces of TiCCDC-1000 film $(6.0 \mathrm{mg})$ soaked with different volumes of $\mathrm{NEt}_{4} \mathrm{BF}_{4} / \mathrm{ACN}$ electrolyte. At a low loading volume of $2.0 \mu \mathrm{L}$ a single broad peak is observed at $-4.0 \mathrm{ppm}$. When the loading volume is increased to $5.0 \mu \mathrm{L}$, this first peak grows in intensity and shifts to higher frequency, whilst a second peak appears

a)
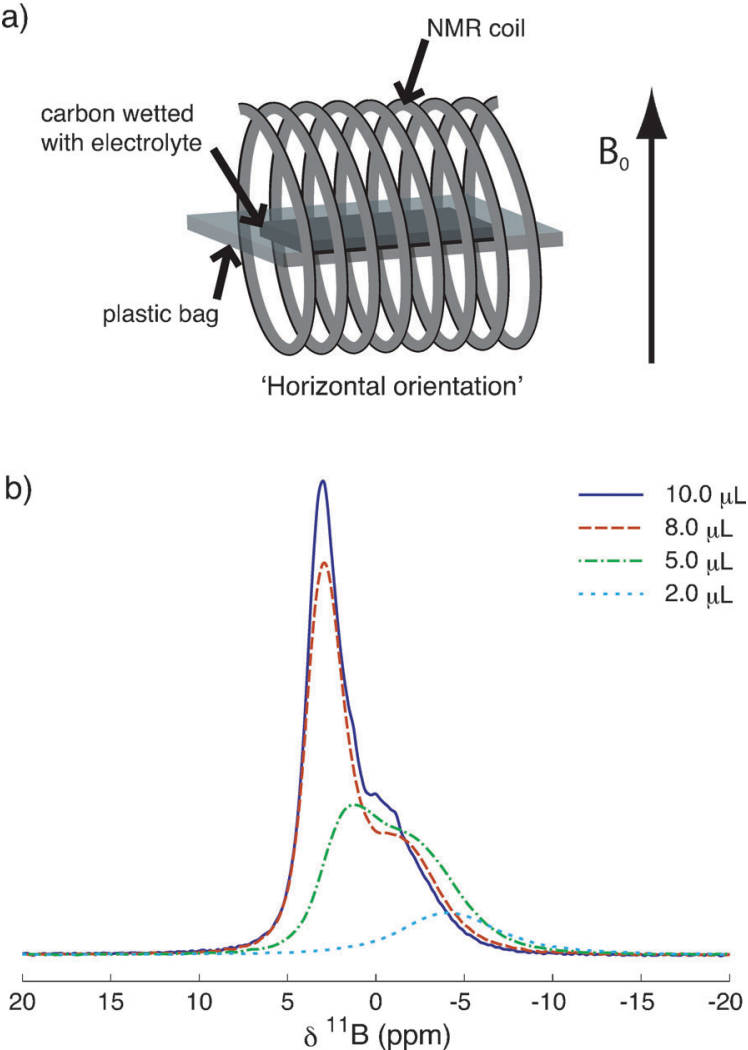

Fig. 1 (a) The experimental set up used to acquire NMR spectra of samples in bags at the horizontal orientation. The applied magnetic field, $B_{0}$, is shown. (b) Static ${ }^{11} B(9.4$ T) NMR spectra of pieces of TiC-CDC-1000 film soaked with different volumes of $\mathrm{NEt}_{4} \mathrm{BF}_{4} / \mathrm{ACN}$ electrolyte (see legend), acquired in the horizontal orientation. Each spectrum is the result of coadding 1024 transients, separated by a recycle interval of $10 \mathrm{~s}$. 
at $1.3 \mathrm{ppm}$. Addition of further electrolyte causes the peak at higher frequency to grow in intensity considerably, whilst the low frequency peak shows only small gains in intensity. For the highest loading volume of $10.0 \mu \mathrm{L}$, an additional small feature is observed at $\sim 0.1 \mathrm{ppm}$.

The different relative growths of the two peaks as a function of loading volume provides an indication of their origin. The approximately constant intensity of the low frequency feature above loading volumes of $5.0 \mu \mathrm{L}$ suggests that this environment corresponds to anions in adsorption sites that are becoming saturated. Thus, the peak at low frequencies $(-4.0 \mathrm{ppm}$ for the $2.0 \mu \mathrm{L}$ sample) is assigned to $\mathrm{BF}_{4}$ anions that are adsorbed to the carbon surface. ${ }^{35}$ Since TiC-CDC is predominantly microporous (see ESI, $\uparrow$ Table S1 and Fig. S2) this peak can be more specifically assigned to anions adsorbed to the carbon inside the micropores, and is referred to as 'in-pore' in our study. As the in-pore sites begin to saturate, anions are forced to occupy positions in large reservoirs between the carbon particles in the film (see ESI, $\uparrow$ Fig. S3), giving rise to the feature at higher frequencies (1.3 ppm for the $5.0 \mu \mathrm{L}$ sample), referred to as 'ex-pore'. Our previous work on an activated carbon ${ }^{35}$ proposed that this feature was due to anions either in the diffuse outer layer of the double layers or in larger pores. In TiC-CDC-1000, there is not space for diffuse layer formation in the pores considering that the diameter of fully-solvated $\mathrm{BF}_{4}{ }^{-}$is $1.16 \mathrm{~nm}^{36}$ and the average pore size in TiC-CDC-1000 is $0.93 \mathrm{~nm}$ (see ESI, $\uparrow$ Table S1). The relatively broad line width of this resonance suggests that it does not correspond to a completely free liquid, but rather to a confined liquid. Thus, this peak must arise from anions confined in spaces between the primary particles. Finally, as the ex-pore sites become saturated, the carbon film can hold no further electrolyte. Therefore, the small feature at $\sim 0.1 \mathrm{ppm}$ in the $10.0 \mu \mathrm{L}$ sample is assigned to $\mathrm{BF}_{4}$ anions in pockets of liquid between the carbon film and the plastic bag, referred to as 'free-electrolyte'. Indeed we observed this feature previously. ${ }^{35}$ The in-pore peak is seen to shift to higher frequencies on the addition of more electrolyte, this observation is discussed later.

Our assignments of the features in Fig. 1b are similar to those in other NMR work on the adsorption of molecules on activated carbons. ${ }^{25-28}$ In particular, one work on the adsorption of hydrogen gas on activated carbons assigned low and high frequency features to hydrogen adsorbed in the micropores and hydrogen in large intergranular pores. ${ }^{28}$ Parallels can also be drawn with work on the adsorption of molecules inside carbon nanotubes, which have assigned similar low and high frequency features to endohedral sites (inside the CNTs) and exohedral sites (outside the CNTs), respectively. ${ }^{29-32}$ Regardless of the nucleus studied, and whether the adsorbate is in the liquid or gaseous state, species adsorbed on carbon show a shift to low frequencies (of between 3 and $12 \mathrm{ppm}$ for different carbons) from the corresponding non-adsorbed resonance. This suggests the chemical shift of adsorbed species is dominated by the influence of the carbon. The shift of the in-pore signal to low frequencies can be attributed to aromatic ring current effects associated with the graphene-like layers in the carbon. ${ }^{37}$
A molecule situated above an aromatic ring or a graphene-like layer is expected to exhibit a shift to low frequencies. Calculations of the chemical shift of molecules adsorbed inside CNTs have previously explored this phenomenon. ${ }^{38,39}$

\section{The effect of carbon pore size on ion adsorption}

Further studies were carried out on TiC-CDC-800 and TiC-CDC600 to investigate the effect of the average pore size on the adsorption of electrolyte species. Fig. 2 shows the static ${ }^{11} \mathrm{~B}$ NMR spectra as well as porosity data for CDC samples studied in this work. Spectra were acquired in the same way as for TiC-CDC1000 (Fig. 1b) to allow comparison. The spectra for TiC-CDC-800 (Fig. 2a) have the same qualitative form as for TiC-CDC-1000 (Fig. 1b). At the lowest loading volume of $2.0 \mu \mathrm{L}$ the in-pore peak is observed at $-4.0 \mathrm{ppm}$. As the loading level is increased to $5.0 \mu \mathrm{L}$ the ex-pore peak is observed at $-0.4 \mathrm{ppm}$. At the highest loading volume of $10.0 \mu \mathrm{L}$ an additional peak assigned to free electrolyte is observed at $0.0 \mathrm{ppm}$. Interestingly, the addition of just $2.0 \mu \mathrm{L}$ of electrolyte appears to effectively saturate the pores. This is in contrast to TiC-CDC-1000 (Fig. 1b) where the in-pore peak grows considerably on the addition of further electrolyte. For TiC-CDC-600 (Fig. 2b) the in-pore peak is visible at $-5.3 \mathrm{ppm}$ for the $2.0 \mu \mathrm{L}$ sample. For higher loading volumes the ex-pore peak dominates the spectrum $(-1.2 \mathrm{ppm}$ for the $5.0 \mu \mathrm{L}$ sample) and the in-pore feature is visible only as a broad shoulder. At the highest loading volume $(15.0 \mu \mathrm{L})$ sharp free electrolyte features are observed at $1.4,1.0$ and $0.6 \mathrm{ppm}$.

The differences in the adsorption spectra for TiC-CDC-1000, -800 and -600 can be rationalised by considering the porosities of the different carbons and the size of the $\mathrm{BF}_{4}$ anion. Fig. $2 \mathrm{c}$ compares the sizes of solvated and desolvated $\mathrm{BF}_{4}{ }^{-}$to the average and maximum pore sizes for the different carbons studied in this work. For TiC-CDC-1000, the average pore size $\left(d_{50}\right)$ is quite similar to the solvated anion size and the maximum pore size is significantly larger, therefore many pores are accessible for adsorption. For TiC-CDC-800, the maximum pore size is approximately equal to the solvated ion size, and the average pore size is smaller, therefore fewer pores are accessible to the anions. This is reflected by the minimal increase in intensity of the in-pore peak on the addition of increasing volumes of electrolyte. Finally, for TiC-CDC-600, both the maximum and average pore sizes are significantly smaller than the solvated anion size and so few pores are accessible to the anions when there is no electrical potential applied to the sample. Consistent with this, the in-pore feature is hard to discern at most loading volumes. These results provide further evidence for our in- and ex-pore peak assignments, identified for TiC-CDC-1000 previously (Fig. 1b). It is interesting to note that in the absence of an applied voltage, few anions are adsorbed on TiC-CDC-600, yet electrochemical studies show this carbon to display the highest capacitance of the three carbons studied here. ${ }^{11,12}$ This illustrates that the electrosorption of ions must be related to (partial) desolvation once an electrical potential is applied. Indeed, the results suggest that ion desolvation does not play a significant role in the 
a) TiC-CDC-800

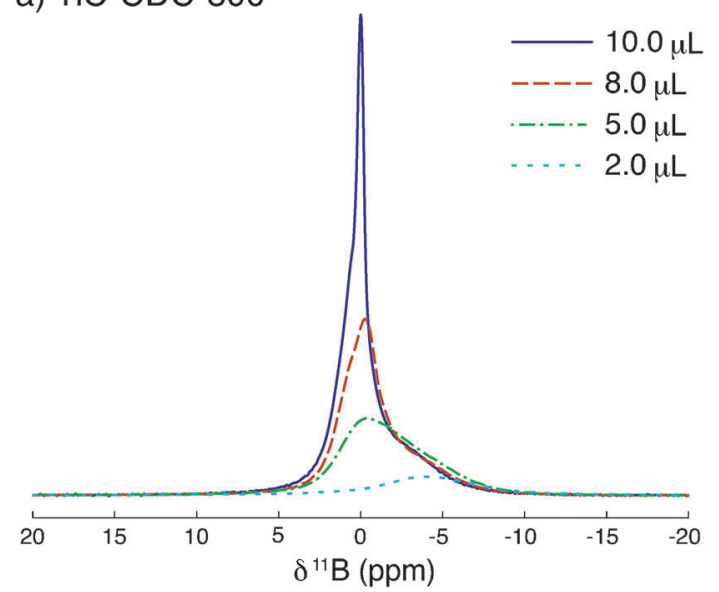

b) TiC-CDC-600

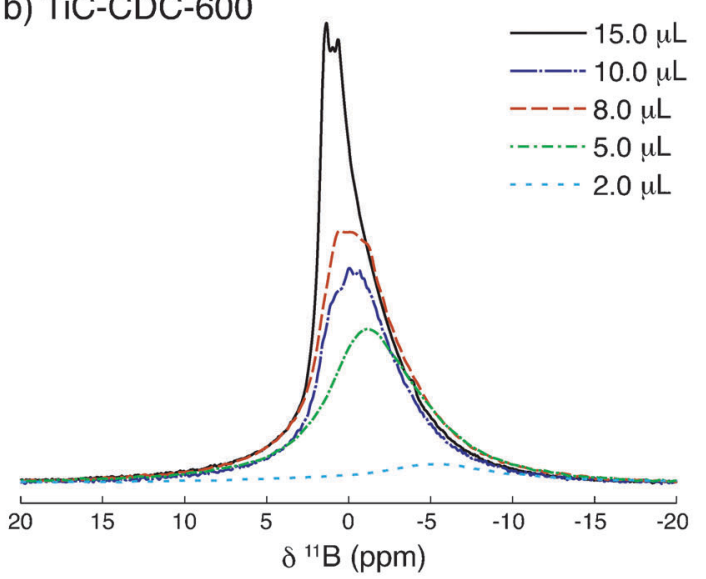

c)

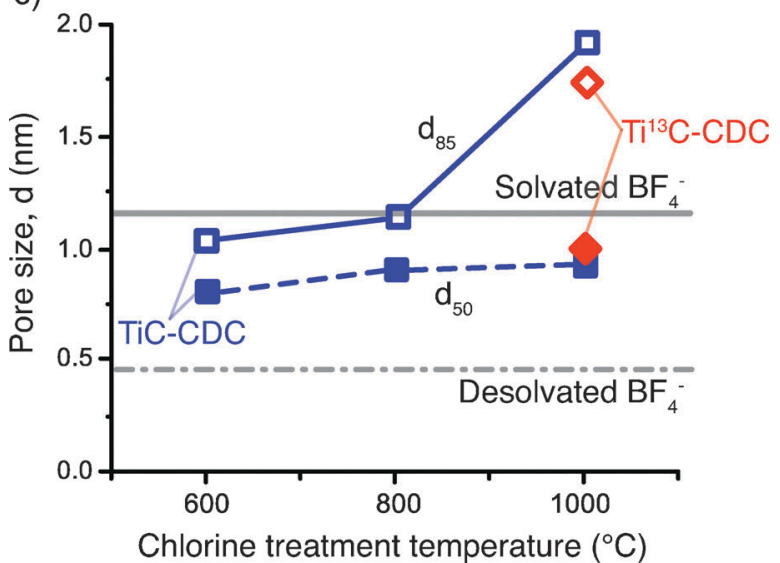

Fig. 2 Static ${ }^{11} \mathrm{~B}(9.4 \mathrm{~T}) \mathrm{NMR}$ spectra of pieces of TiC-CDC film soaked with different volumes of $\mathrm{NEt}_{4} \mathrm{BF}_{4} / \mathrm{ACN}$ electrolyte. Spectra are shown for (a) TiC-CDC-800 and (b) TiC-CDC-600. Each spectrum is the result of coadding 1024 transients, separated by a recycle interval of $10 \mathrm{~s}$. (c) TiC-CDC pores sizes for carbons considered in this work as well as solvated and desolvated anion sizes ${ }^{36}$ are shown. $d_{n}$ is the pore size where $n \%$ of the pore volume is below that size such that $d_{50}$ is the average pore size ( $d_{85}$ is referred to as the maximum pore size).

adsorption process in the absence of an applied voltage; if it did, essentially all of the carbon pores would be accessible to the anions for all three carbons studied.

\section{The effect of sample orientation on NMR spectra}

Recent NMR studies of systems contained within plastic bags ${ }^{40}$ and between flat plates ${ }^{41,42}$ have noted significant effects on the observed shift associated with the orientation of the sample with respect to the applied magnetic field. These effects have been attributed to bulk magnetic susceptibility (BMS) effects arising from the highly inhomogeneous shape of the sample. To investigate this effect, a TiC-CDC-1000 sample $(6.0 \mathrm{mg})$ loaded with $\mathrm{NEt}_{4} \mathrm{BF}_{4} / \mathrm{ACN}$ electrolyte $(10.0 \mu \mathrm{L})$ was studied in two different orientations, horizontal and vertical (Fig. 3a and b).

${ }^{11} \mathrm{~B}$ static NMR spectra are shown in Fig. 3c. As anticipated, the orientation of the sample relative to the applied magnetic field has a marked effect on the NMR spectrum. In the vertical orientation peaks are shifted to lower frequencies and better resolution between the in- and ex-pore peak is observed. On changing the sample orientation from horizontal to vertical, the in-pore peak position changes more than the ex-pore peak, with absolute changes of $\sim 10$ and $8 \mathrm{ppm}$ respectively. The small feature attributed to free-electrolyte appears to shift by a noticeably smaller amount of $\sim 2 \mathrm{ppm}$. This indicates that the in-pore, ex-pore and free electrolyte features experience different BMS effects. Crucially, future experiments should carefully consider the effects of sample orientation in the NMR coil. Indeed, this has been shown to be an important consideration in in situ studies of lithium-ion batteries. ${ }^{40}$

In the vertical orientation, increased resolution of the in- and ex-pore features allows for more accurate fitting of the spectra to enable an estimation of the fraction of anions that a)

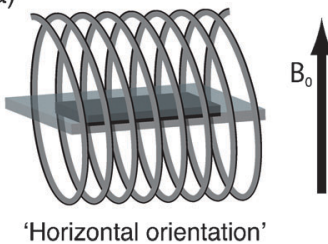

b)

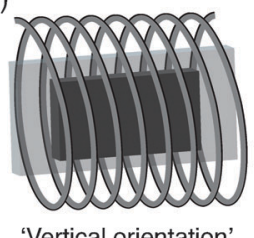

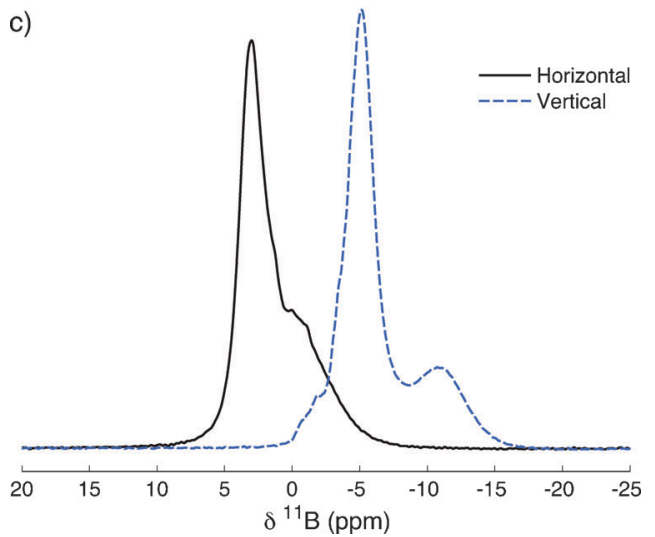

Fig. 3 The horizontal (a) and vertical (b) orientations used to acquire NMR spectra of samples in bags. (c) Static (9.4 T) ${ }^{11} \mathrm{~B}$ NMR spectra of a piece of TiC$\mathrm{CDC}-1000$ film soaked with $10.0 \mu \mathrm{L}$ of $\mathrm{NEt}_{4} \mathrm{BF}_{4} / \mathrm{ACN}$ electrolyte, in the horizontal and vertical orientations. Each spectrum is the result of coadding 1024 transients, separated by a recycle interval of $10 \mathrm{~s}$. 
are adsorbed. A deconvolution was performed assuming a Gaussian line shape for the in-pore environment since a distribution of adsorption sites is expected in the micropores. The line shape corresponding to the ex-pore environment is expected to be close to a Lorentzian owing to its liquid-like form. However, confinement may result in reduced mobility and therefore some Gaussian broadening. Therefore the Gaussian/Lorentzian ratio in the fit was allowed to vary to encapsulate this behaviour. Fitting (see ESI, $\uparrow$ Fig. S4) reveals that $\sim 21 \%$ of the total number of anions in the sample are adsorbed in the pores. Taking the specific surface area for TiC-CDC-1000 as $1723 \mathrm{~m}^{2} \mathrm{~g}^{-1}$ (see ESI, $\dagger$ Table S1) and assuming a monolayer of adsorbed anions on a planar surface, the area containing one $\mathrm{BF}_{4}$ anion is calculated to be $5.4 \mathrm{~nm}^{2}$ (see ESI $\dagger$ for details). This is comparable to the theoretical lower limit of $3.0 \mathrm{~nm}^{2}$ per anion, calculated for a close packed square lattice (see ESI, $\uparrow$ Fig. S5) of solvated anions and cations with sizes ${ }^{36}$ of 1.16 and $1.30 \mathrm{~nm}$ respectively, the larger effective footprint of the anion being expected due to the curvature of the internal carbon surfaces.

\section{4. ${ }^{19}$ F NMR - a further probe of anion adsorption}

A ${ }^{19}$ F NMR adsorption study was carried out on TiC-CDC-1000 to probe the adsorption of the $\mathrm{BF}_{4}$ anions in more detail. $\mathrm{A}$ preliminary experiment showed the NMR spectra to have a very similar orientation dependence to that shown in Fig. 3, with better resolution of peaks in the vertical orientation (see ESI, $\dagger$ Fig. S6). Fig. 4 shows the static ${ }^{19} \mathrm{~F}$ spectra of samples in bags containing pieces of TiC-CDC-1000 film $(6.0 \mathrm{mg})$ soaked with different volumes of $\mathrm{NEt}_{4} \mathrm{BF}_{4} / \mathrm{ACN}$ electrolyte, acquired in the vertical orientation. The ${ }^{19} \mathrm{~F}$ spectra reveal in- and ex-pore environments for $\mathrm{BF}_{4}{ }^{-}$, consistent with the ${ }^{11} \mathrm{~B}$ spectra (Fig. 1b). Moreover, the relative growth of these features as a function of loading volume is very similar to that seen in the ${ }^{11} \mathrm{~B}$ experiment. At a low loading volume of $2.0 \mu \mathrm{L}$ the in-pore peak is observed at -156.4 ppm (Fig. 4). When the loading volume is increased to $5.0 \mu \mathrm{L}$ the ex-pore peak begins to become visible at

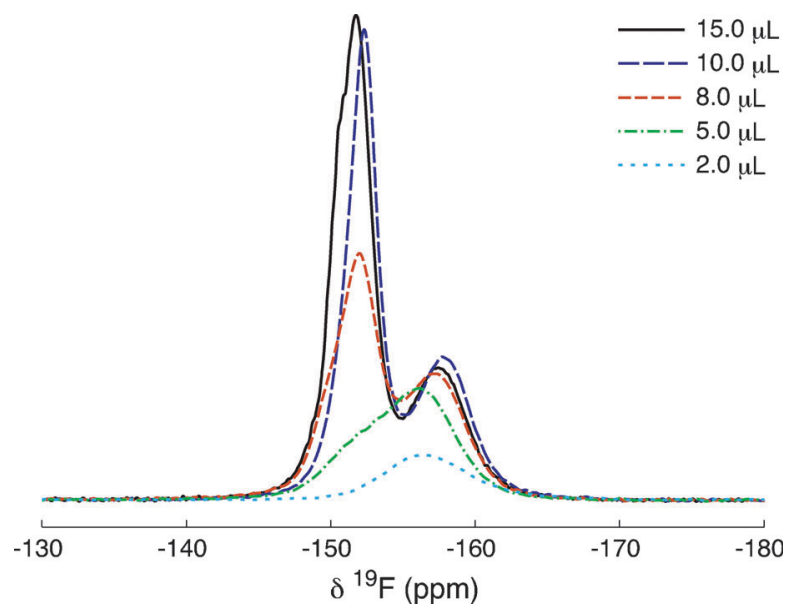

Fig. 4 Static (7.05 T) ${ }^{19} \mathrm{~F}$ NMR spectra of TiC-CDC-1000 film pieces $(6.0 \mathrm{mg})$ soaked with different volumes of $\mathrm{NEt}_{4} \mathrm{BF}_{4} / \mathrm{ACN}$ electrolyte, acquired in the vertical orientation. Each spectrum is the result of coadding 64 transients, separated by a recycle interval of $10 \mathrm{~s}$. higher frequency. Addition of further electrolyte causes the in-pore environment to shift to lower frequencies and to saturate, whilst the ex-pore environment shows a considerable increase in intensity. For the highest loading volume of $15.0 \mu \mathrm{L}$ a shoulder attributed to free electrolyte (between the carbon film and the plastic bag) is observed at $-\mathbf{1 5 0 . 6} \mathrm{ppm}$. Fitting of the $10.0 \mu \mathrm{L}$ spectrum (see ESI, $\uparrow$ Fig. S4) reveals that $\sim 26 \%$ of the anions in the sample are adsorbed in the pores. Assuming a monolayer of adsorbed anions on a planar surface, the area containing one $\mathrm{BF}_{4}$ anion is calculated to be $4.4 \mathrm{~nm}^{2}$. This is in good agreement with the value of $5.4 \mathrm{~nm}^{2}$ obtained above for the corresponding ${ }^{11} \mathrm{~B}$ spectrum. The small difference between these two values likely arises from the experimental errors associated with weighing the carbon and syringing the electrolyte in the fabrication of the two different samples used in these measurements. We note an apparent shift for the in-pore peak to lower frequencies on loading with increased volumes of electrolyte. The opposite trend is observed for spectra of the same samples acquired in the horizontal orientation (see ESI, $\dagger$ Fig. S7), suggesting that this shift is due to a BMS effect.

The ${ }^{19} \mathrm{~F}$ and ${ }^{11} \mathrm{~B}$ spectra lead us to the same conclusion, namely, that there are two main environments for the anions inside the carbon film. This is consistent with the assertion that the chemical shift of the in-pore peak arises from the carbon, and that adsorption is physical and not chemical in nature. The separation of the in-pore and ex-pore environments is very similar for ${ }^{11} \mathrm{~B}$ and ${ }^{19} \mathrm{~F}$, measured as $5.7 \mathrm{ppm}$ and $5.5 \mathrm{ppm}$ respectively for the $10.0 \mu \mathrm{L}$ samples in the vertical orientation. This suggests that the fluorine and boron atoms experience similar shielding effects from the carbon.

\section{Comparison between anion and cation adsorption}

To probe the $\mathrm{NEt}_{4}$ cation environments, ${ }^{1} \mathrm{H}$ NMR experiments were performed on TiC-CDC-1000 in plastic bags (see ESI, $\dagger$ Fig. S8). Whilst spectra show qualitative agreement with studies of the $\mathrm{BF}_{4}$ anions, with both in- and ex-pore features, they are complicated by the ${ }^{1} \mathrm{H}$ signal from the plastic bag used to contain the sample. To circumvent this issue associated with samples in bags and to allow direct comparison of the anion and cation environments, ${ }^{19} \mathrm{~F}$ and ${ }^{1} \mathrm{H}$ experiments were performed on samples in MAS rotors. A MAS probe was used for the experiments and the spectra were acquired in static mode, the sample orientation corresponding to the magic-angle (MA; 54.74 ${ }^{\circ}$. Fig. 5 shows ${ }^{19} \mathrm{~F}$ and ${ }^{1} \mathrm{H}$ NMR spectra of TiCCDC-1000 (3.0 mg) samples in MAS rotors soaked with different volumes of $\mathrm{NEt}_{4} \mathrm{BF}_{4} / \mathrm{dACN}$ electrolyte. Static ${ }^{19} \mathrm{~F}$ (Fig. 5a) and ${ }^{1} \mathrm{H}$ spectra (Fig. 5b) are acquired on the same samples, and spectra of the neat electrolyte are also overlaid.

Whilst some resolution is lost at the MA sample orientation due to negligible BMS effects (compared to Fig. 4), the same general trends seen in the previous spectra (Fig. 1b and 4) are reproduced. In both ${ }^{19} \mathrm{~F}$ and ${ }^{1} \mathrm{H}$ spectra, only the in-pore peak is seen at a loading volume of $1.0 \mu \mathrm{L}$, and the ex-pore environment appears for the $2.5 \mu \mathrm{L}$ sample. Further addition of electrolyte causes only small increases in intensity of the in-pore environment as it begins to saturate, whilst the ex-pore 

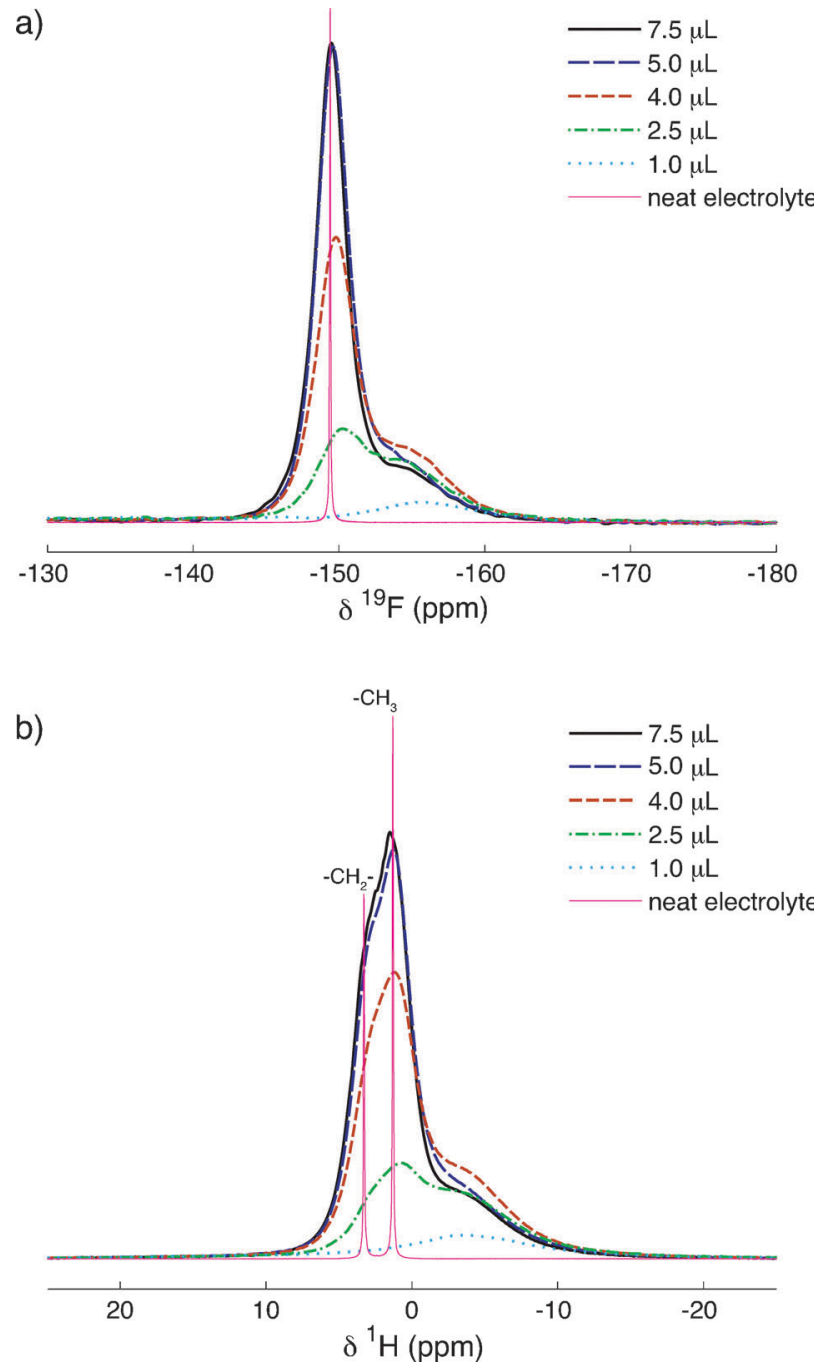

Fig. 5 (a) ${ }^{19} \mathrm{~F}$ and (b) ${ }^{1} \mathrm{H}$ NMR (7.05 T) spectra of TiC-CDC-1000 (3.0 mg) soaked with different volumes of $\mathrm{NEt}_{4} \mathrm{BF}_{4} / \mathrm{dACN}$ electrolyte in MAS rotors, acquired in static mode at the magic-angle. The same carbon mass to electrolyte volume ratios were used as for the experiments on samples in bags. ${ }^{19} \mathrm{~F}$ and ${ }^{1} \mathrm{H}$ spectra are shown for the same samples, allowing for direct comparison of the anion and cation environments. The spectrum of free electrolyte is also shown for comparison; the $-\mathrm{CH}_{2}$ and $-\mathrm{CH}_{3}$ resonances are labelled in the ${ }^{1} \mathrm{H}$ spectrum. Each ${ }^{19} \mathrm{~F}$ spectrum is the result of coadding 16 transients with a recycle interval of $15 \mathrm{~s}$, whilst for each ${ }^{1} \mathrm{H}$ spectrum 32 transients and a recycle interval of $8 \mathrm{~s}$ were used.

peak increases in intensity considerably. Since the $\mathrm{NEt}_{4}$ cations have a $-\mathrm{CH}_{2}-$ and a $-\mathrm{CH}_{3}$ group, two resonances are seen for the ex-pore environment (and neat electrolyte), the $-\mathrm{CH}_{2}-$ resonance appearing at higher frequency. Interestingly, the ex-pore environments show very similar resonant frequencies to the neat electrolyte peaks.

Careful NMR studies of planar lithium-ion battery electrodes have shown that BMS effects are minimised when samples are orientated at the MA, ${ }^{40}$ signals for species within the electrodes giving rise to resonances with shifts close to their isotropic values obtained under MAS. This phenomenon could be rationalised by considering the (long-range) dipolar interactions that occur between nuclear spins and the magnetic moments of the nearby particles within the electrode films, the orientation dependent shifts being significant when paramagnetic and conducting (carbon and metal) particles were present. The overlap of the neat electrolyte and ex-pore peaks in our system at the MA (Fig. 5) indicates that the shift of the latter feature is largely dominated by BMS (long-range) interactions. This overlap adds further support to our assignment of the ex-pore species being located between carbon particles without any significant adsorption occurring. While the resonant frequency of the in-pore feature is also affected by BMS effects, a substantial contribution to its shift must arise from a local field interaction, that is from the nearby ring currents of the carbon. ${ }^{27,28,37}$ We note that the $-\mathrm{CH}_{2}-$ and $-\mathrm{CH}_{3}$ resonances are not resolvable for the in-pore environment due to their broad shape.

The key observation from Fig. 5 is that the ${ }^{19} \mathrm{~F}$ and ${ }^{1} \mathrm{H}$ spectra have an almost identical form. This indicates that the adsorption behaviour of anions and cations is the same in the absence of an applied voltage. For systems with a point of zero charge close to $\mathrm{pH} 7.0$, this behaviour is expected if charge neutrality is to be maintained in the solution and the carbon. Indeed this has been observed in molecular dynamics simulations of an ionic liquid. ${ }^{16}$ It is noted that spectra of the 5.0 and $7.5 \mu \mathrm{L}$ samples have a very similar form, the resonances from the $7.5 \mu \mathrm{L}$ sample being only marginally more intense. This behaviour is attributed to loss of electrolyte during microsyringing of the larger volume. We also note that the $4.0 \mu \mathrm{L}$ sample has a larger in-pore occupation than the other samples, though we do not offer an explanation for this deviation.

\section{Through-space magnetisation transfer experiment}

Our NMR experiments on CDCs soaked with different volumes of electrolyte provide insight into the nature of the observed resonances and strongly indicate the presence of two distinct ion environments. However the resolution of these environments remains limited. For future experiments, it would be advantageous to observe just the in-pore environment; that is ions adsorbed on the carbon surface in the micropores. Cross polarisation (CP) is routinely used in NMR for magnetisation transfer over short distances. ${ }^{43}$ It is typically used to achieve a signal enhancement, but can also be used for spectral editing so as to favour species close to carbon (or other nuclei). ${ }^{44-47}$ In view of this, CDCs enriched with the NMR-active ${ }^{13} \mathrm{C}$ isotope (99\% enrichment) were prepared.

Fig. 6 shows the static direct excitation and ${ }^{13} \mathrm{C} \rightarrow{ }^{1} \mathrm{H} \mathrm{CP}$ spectra of a $\mathrm{Ti}^{13} \mathrm{C}-\mathrm{CDC}-1000(3.0 \mathrm{mg})$ sample in a MAS rotor with $7.5 \mu \mathrm{L} \mathrm{NEt}_{4} \mathrm{BF}_{4} / \mathrm{dACN}$ electrolyte. This loading volume was chosen since the direct excitation spectrum is expected to show both in- and ex-pore peaks (Fig. 5b). Indeed, direct excitation gives a very similar spectrum to that for natural abundance TiCCDC-1000. In contrast, the ${ }^{13} \mathrm{C} \rightarrow{ }^{1} \mathrm{H}$ CP experiment reveals solely the in-pore environment. This confirms that these cations are spatially close to the carbon, since magnetisation transfer is by dipolar coupling, a short range through-space interaction. This observation confirms our peak assignments. Indeed the absence of ex-pore peaks confirms that these 


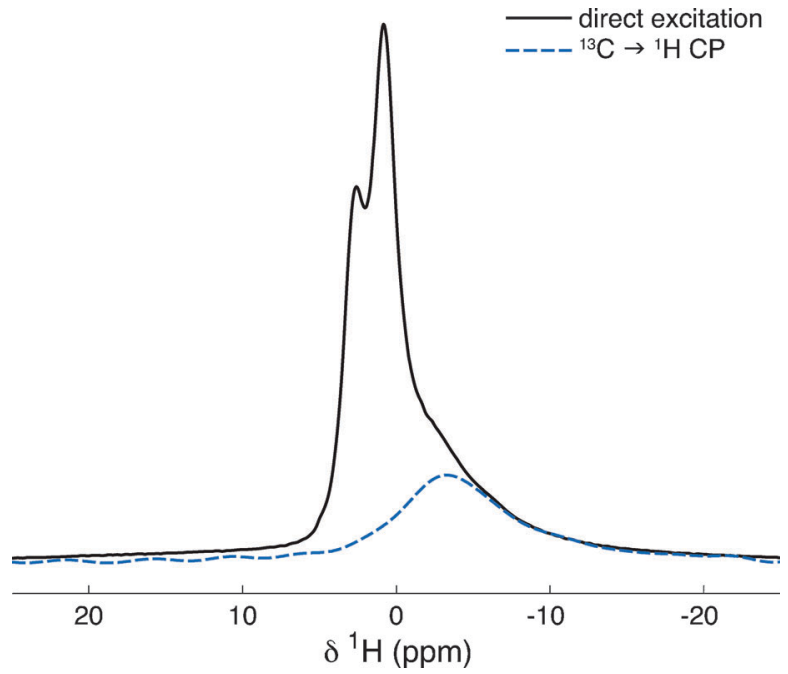

Fig. 6 Direct excitation and ${ }^{13} \mathrm{C} \rightarrow{ }^{1} \mathrm{H}$ CP NMR spectra (7.05 T) of TiC-CDC-1000 (3.0 mg) soaked with $7.5 \mu \mathrm{L}$ of $\mathrm{NEt}_{4} \mathrm{BF}_{4} / \mathrm{dACN}$ electrolyte in a MAS rotor, acquired in static mode at the magic-angle. CP only shows protons (in the cations) that are spatially close to the carbon, confirming that these (in-pore) cations are adsorbed to the surface. The direct excitation and CP spectra are the result of coadding 32 and 4608 transients respectively, transients separated by a recycle interval of $3 \mathrm{~s}$. Spectra are scaled for ease of comparison. The pulse sequence for the $\mathrm{CP}$ experiment is given in the ESIt (Fig. S9).

cations are further from the carbon than the in-pore cations. We note that in the ${ }^{13} \mathrm{C} \rightarrow{ }^{1} \mathrm{H} \mathrm{CP}$ spectrum it is still not possible to resolve the in-pore $-\mathrm{CH}_{2}-$ and $-\mathrm{CH}_{3}$ resonances, as the overall line width is much bigger than the expected separation of these resonances $(\sim 2 \mathrm{ppm})$. Whilst other $\mathrm{CP}$ contact times (the time during which magnetisation is transferred) were studied, we only present the spectrum for the longest time $(8 \mathrm{~ms})$ here, since it gave the greatest signal intensity.

The ${ }^{13} \mathrm{C} \rightarrow{ }^{1} \mathrm{H}$ CP experiment could be applied to study adsorption on other carbon materials, both to provide more definitive peak assignments as well as to resolve peaks from adsorbed and non-adsorbed species. For example, it could confirm whether resonances assigned to molecules located in the exohedral sites of carbon nanotubes ${ }^{29,30}$ are adsorbed to the exterior of the CNTs or not. The technique could also be used to confirm the presence of layered interfaces, such as that proposed for methanol gas inside large CNTs where one layer is adsorbed to the carbon and the other is in the centre of the nanotube. ${ }^{29}$ The ${ }^{13} \mathrm{C} \rightarrow{ }^{1} \mathrm{H}$ CP experiment could help resolve the in-pore peak when features overlap, as in ${ }^{1} \mathrm{H}$ NMR spectra of hydrogen gas adsorbed on activated carbons with large pore sizes. ${ }^{28}$

\section{Conclusions}

This work has presented a systematic NMR study of electrolyte adsorption on TiC-CDCs, a model carbon morphology used as a supercapacitor electrode material. The experiments have provided fundamental insight into the different electrolyte ion environments present in the carbon. In-pore features appear at lower NMR frequencies and arise from ions adsorbed in the micropores of the carbon, while ex-pore features arise from free ions in the spaces between carbon particles. These environments have been studied as a function of pore size, the results corroborating our spectral assignments. For TiC-CDC-600, the carbon with the smallest pore size studied, few anions were adsorbed in the micropores as a large fraction of the total pore volume is virtually inaccessible to solvated anions. Multinuclear NMR experiments point towards identical adsorption behaviour for anions and cations.

Our work highlights some of the experimental considerations that will be key to in situ studies of supercapacitors. The effects of sample orientation have been explored and ${ }^{13} \mathrm{C} \rightarrow{ }^{1} \mathrm{H}$ CP NMR experiments, with ${ }^{13} \mathrm{C}$-enriched carbons, have been developed to select the ions adsorbed in the pores. While careful choice of sample orientation can improve spectral resolution, $\mathrm{CP}$ experiments allow selection of just the adsorbed species in the NMR spectrum. This is highly desirable since it is these ions that contribute to the charge storage in supercapacitors. A combination of the techniques presented here will be of great use in envisaged in situ experiments as well as experiments in the broader field of adsorption on porous carbons.

\section{Acknowledgements}

ACF, JMG, HW and CPG acknowledge the Sims Scholarship (ACF), EPSRC and the EU ERC for funding. ACF and JMG thank the NanoDTC (Cambridge) for travel funding. NMT was supported by Northeastern Center for Chemical Energy Storage, an Energy Frontier Research Center funded by U.S. Department of Energy, Office of Science, Office of Basic Energy Sciences under Award \#DE-SC0001294. VP acknowledges funding from the German Federal Ministry for Research and Education (BMBF) in support of the nanoEES ${ }^{3 \mathrm{D}}$ project (award number 03EK3013) as part of the strategic funding initiative energy storage framework. YG was supported by the U.S. Department of Energy, Office of Basic Energy Sciences, Division of Materials Sciences and Engineering, under Award No. DE-FG02-07ER46473. PS acknowledges funding from the European Research Council (ERC, Advanced Grant, ERC2011-AdG, Project 291543 - IONACES). The authors are grateful to Mohamed Shamma for help with the synthesis of isotopic TiC, Boris Dyatkin for his help with gas sorption analysis and synthesis of isotopic CDC, and Min Heon for his support with scanning electron microscopy (all at Drexel University). Dr Mesut Aslan (INM) is thanked for his help with gas sorption analysis. VP thanks Prof. Eduard Arzt for his continuing support.

\section{Notes and references}

1 J. R. Miller and P. Simon, Science, 2008, 321, 651-652.

2 M. Winter and R. Brodd, Chem. Rev., 2004, 104, 4245-4269.

3 J. R. Miller and A. Burke, Electrochem. Soc. Interface, 2008, 17, 53-57.

4 Y. Zhai, Y. Dou, D. Zhao, P. F. Fulvio, R. T. Mayes and S. Dai, Adv. Mater., 2011, 23, 4828-4850.

5 A. Burke, Electrochim. Acta, 2007, 53, 1083-1091.

6 A. G. Pandolfo and A. F. Hollenkamp, J. Power Sources, 2006, 157, 11-27. 
7 E. Frackowiak, Phys. Chem. Chem. Phys., 2007, 9, 1774-1785. 8 V. Presser, M. Heon and Y. Gogotsi, Adv. Funct. Mater., 2011, 21, 810-833.

9 G. Laudisio, R. K. Dash, J. P. Singer, G. Yushin, Y. Gogotsi and J. E. Fischer, Langmuir, 2006, 22, 8945-8950.

10 R. Dash, J. Chmiola, G. Yushin, Y. Gogotsi, G. Laudisio, J. Singer, J. Fischer and S. Kucheyev, Carbon, 2006, 44, 2489-2497.

11 J. Chmiola, G. Yushin, Y. Gogotsi, C. Portet, P. Simon and P. L. Taberna, Science, 2006, 313, 1760-1763.

12 J. Chmiola, C. Largeot, P. L. Taberna, P. Simon and Y. Gogotsi, Angew. Chem., Int. Ed., 2008, 47, 3392-3395.

13 P. Simon and Y. Gogotsi, Acc. Chem. Res., 2012, DOI: 10.1021/ar200306b.

14 M. V. Fedorov and A. A. Kornyshev, Electrochim. Acta, 2008, 53, 6835-6840.

15 J. C. Palmer, A. Llobet, S.-H. Yeon, J. E. Fischer, Y. Shi, Y. Gogotsi and K. E. Gubbins, Carbon, 2010, 48, 1116-1123.

16 C. Merlet, B. Rotenberg, P. A. Madden, P. L. Taberna, P. Simon, Y. Gogotsi and M. Salanne, Nat. Mater., 2012, 11, 306-310.

17 S. Kondrat and A. Kornyshev, J. Phys.: Condens. Matter, 2011, 23, 022201.

18 S. Kondrat, N. Georgi, M. V Fedorov and A. A. Kornyshev, Phys. Chem. Chem. Phys., 2011, 13, 11359-11366.

19 S. Kondrat, C. R. Pérez, V. Presser, Y. Gogotsi and A. A. Kornyshev, Energy Environ. Sci., 2012, 5, 6474-6479.

20 R. Lin, P. L. Taberna, J. Chmiola, D. Guay, Y. Gogotsi and P. Simon, J. Electrochem. Soc., 2009, 156, A7-A12.

21 T. M. Arruda, M. Heon, V. Presser, P. C. Hillesheim, S. Dai, Y. Gogotsi, S. V. Kalinin and N. Balke, Energy Environ. Sci., 2013, 6, 225-231.

22 M. D. Levi, G. Salitra, N. Levy, D. Aurbach and J. Maier, Nat. Mater., 2009, 8, 872-875.

23 S. Boukhalfa, L. He, Y. B. Melnichenko and G. Yushin, Angew. Chem., Int. Ed., 2013, 52, 1-6.

24 M. D. Levi, S. Sigalov, G. Salitra, R. Elazari, D. Aurbach, L. Daikhin and V. Presser, J. Phys. Chem. C, 2013, 117, 1247-1256.

25 R. K. Harris, T. V. Thompson, P. R. Norman, C. Pottage and A. N. Trethewey, J. Chem. Soc., Faraday Trans., 1995, 91, 1795-1799.

26 R. K. Harris, T. V. Thompson, P. R. Norman and C. Pottage, Carbon, 1999, 37, 1425-1430.
27 L. M. Dickinson, R. K. Harris, J. A. Shaw, M. Chinn and P. R. Norman, Magn. Reson. Chem., 2000, 38, 918-924.

28 R. J. Anderson, T. P. McNicholas, A. Kleinhammes, A. Wang, J. Liu and Y. Wu, J. Am. Chem. Soc., 2010, 132, 8618-8626.

29 X. Liu, X. Pan, W. Shen, P. Ren, X. Han and X. Bao, J. Phys. Chem. C, 2012, 116, 7803-7809.

30 W. Sekhaneh, M. Kotecha, U. Dettlaff-Weglikowska and W. S. Veeman, Chem. Phys. Lett., 2006, 428, 143-147.

31 Q. Chen, J. L. Herberg, G. Mogilevsky, H.-J. Wang, M. Stadermann, J. K. Holt and Y. Wu, Nano Lett., 2008, 8, 1902-1905.

32 K. Shen and T. Pietrass, J. Phys. Chem. B, 2004, 108, 9937-9942.

33 S.-I. Lee, K. Saito, K. Kanehashi, M. Hatakeyama, S. Mitani, S.-H. Yoon, Y. Korai and I. Mochida, Carbon, 2006, 44, 2578-2586.

34 M. Deschamps, E. Gilbert, P. Azais, E. Raymundo-Piñero, M. R. Ammar, P. Simon, D. Massiot and F. Béguin, Nat. Mater., 2013, 12, 351-358.

35 H. Wang, T. K.-J. Köster, N. M. Trease, J. Ségalini, P.-L. Taberna, P. Simon, Y. Gogotsi and C. P. Grey, J. Am. Chem. Soc., 2011, 133, 19270-19273.

36 Y.-J. Kim, Y. Masutzawa, S. Ozaki, M. Endo and M. S. Dresselhaus, J. Electrochem. Soc., 2004, 151, E199-E205.

37 P. Lazzeretti, Prog. Nucl. Magn. Reson. Spectrosc., 2000, 36, $1-88$.

38 N. A. Besley and A. Noble, J. Chem. Phys., 2008, 128, 101102.

39 M. Kibalchenko, M. C. Payne and J. R. Yates, ACS Nano, 2011, 5, 537-545.

40 N. M. Trease, L. Zhou, H. J. Chang, B. Y. Zhu and C. P. Grey, Solid State Nucl. Magn. Reson., 2012, 42, 62-70.

41 R. Ulrich, R. W. Glaser and A. S. Ulrich, J. Magn. Reson., 2003, 164, 115-127.

42 R. W. Glaser and A. S. Ulrich, J. Magn. Reson., 2003, 164, 104-114.

43 D. C. Apperley, R. K. Harris and P. Hodgkinson, Solid-state NMR Basic Principles and Practice, Momentum Press, 2012.

44 J. Kolmas and W. Kolodziejski, Chem. Phys. Lett., 2012, 554, 128-132.

45 J. Kolmas and W. Kolodziejski, Chem. Commun., 2007, 4390-4392.

46 W. Kolodziejski, A. Corma, K. Wozniak and J. Klinowski, J. Phys. Chem., 1996, 100, 7345-7351.

47 J. L. White, L. W. Beck and J. F. Haw, J. Am. Chem. Soc., 1992, 114, 6182-6189. 